\title{
Eficiencia de la remoción de materia orgánica mediante electrocoagulación para tratar aguas residuales
} procedentes de mataderos

\section{Efficiency of organic matter removal by electrocoagulation to treat wastewater from slaughterhouses}

\author{
Henry Giovani Jave Concepción ${ }^{1 *} \mathbb{D}$, Jesús Rascón ${ }^{2} \mathbb{D}$, Kátterin Jina Luz Pinedo Gómez ${ }^{1} \mathbb{D}$, Francesca Zenith Guzmán \\ Trujillo $^{3}$ (D), Dayan Troya Paredes ${ }^{3}$ (D)
}

\section{RESUMEN}

En los mataderos se genera grandes volúmenes de aguas residuales con alto contenido de materia orgánica, lo cual ocasiona impactos significativos en los cuerpos receptores. Por lo cual, el objetivo de la presente investigación fue evaluar la eficiencia de diferentes procesos de electrocoagulación en la remoción de la materia orgánica presente en el agua residual de un matadero. Para lo cual, se realizaron mediciones antes de la aplicación del sistema de electrocoagulación y después de la aplicación del mencionado sistema. Los resultados explican un grado de confiabilidad del 0,95, indicando que dicho sistema con aplicaciones de $12 \mathrm{Vx} 15 \mathrm{~min}$ (T1) y $24 \mathrm{Vx} 10 \mathrm{~min}$ (T2) $\log$ ran reducir los parámetros de demanda bioquímica de oxígeno (DBO) de $585 \mathrm{mg} / \mathrm{L}$ a $183,83 \mathrm{mg} / \mathrm{L}$ (T1) y $104,17 \mathrm{mg} / \mathrm{L}$ (T2), la turbiedad de 444 UNT a 39,67 UNT (T1) y 26,00 UNT (T2), la conductividad eléctrica de $1579 \mu \mathrm{mho} / \mathrm{cm}$ a $359,40 \mu \mathrm{mho} / \mathrm{cm}$ (T1) y 128,92 $\mu \mathrm{mho} / \mathrm{cm}$ (T2), los sólidos suspendidos totales (SST) de $466 \mathrm{mg} / \mathrm{La} 28,83 \mathrm{mg} / \mathrm{L}$ (T1) y $14,50 \mathrm{mg} / \mathrm{L}(\mathrm{T} 2)$ y el potencial de hidrógeno $(\mathrm{pH})$ desde 11,77 unidades de $\mathrm{pH}$ hasta 8,34 y 7,53 respectivamente; concluyéndose que, existiendo diferencia significativa entre los tratamientos.

Palabras clave: agua residual, electrocoagulación, matadero, parámetros fisicoquímicos.

\begin{abstract}
Slaughterhouses generate large volumes of wastewater with high organic matter content, which causes significant impacts on the receiving bodies. Therefore, the objective of this research was to evaluate the efficiency of different electrocoagulation processes in the removal of organic matter present in the wastewater from a slaughterhouse. For this purpose, measurements were made before and after the application of the electrocoagulation system. The results explain a degree of reliability of 0.95 , indicating that the system with applications of $12 \mathrm{Vx} 15 \mathrm{~min}(\mathrm{~T} 1)$ and $24 \mathrm{Vx} 10 \mathrm{~min}$ (T2) reduced the parameters of biochemical oxygen demand (BOD) from $585 \mathrm{mg} / \mathrm{L}$ to $183.83 \mathrm{mg} / \mathrm{L}$ (T1) and $104.17 \mathrm{mg} / \mathrm{L}$ (T2), turbidity from $444 \mathrm{UNT}$ to $39,67 \mathrm{UNT}$ (T1) and $26.00 \mathrm{UNT}$ (T2), electrical conductivity from $1579 \mu \mathrm{mho} / \mathrm{cm}$ to $359.40 \mu \mathrm{mho} / \mathrm{cm}$ (T1) and $128.92 \mu \mathrm{mho} / \mathrm{cm}$ (T2), total suspended solids (TSS) from $466 \mathrm{mg} / \mathrm{L}$ to $28.83 \mathrm{mg} / \mathrm{L}(\mathrm{T} 1)$ and $14.50 \mathrm{mg} / \mathrm{L}(\mathrm{T} 2)$ and potential hydrogen $(\mathrm{pH})$ from $11.77 \mathrm{pH}$ units to 8.34 and 7.53 respectively; The conclusion was that there was a significant difference between the treatments.
\end{abstract}

Keywords: waste water, electrocoagulation, slaughterhouse, physico-chemical parameters.

\footnotetext{
${ }^{1}$ Corporación GRONPERU SAC., Tarapoto, Perú.

${ }^{2}$ Universidad Nacional Toribio Rodríguez de Mendoza de Amazonas, Instituto de Investigación para el Desarrollo Sustentable de Ceja de Selva, Chachapoyas, Perú

${ }^{3}$ Universidad Peruana Unión, Campus Tarapoto, Tarapoto, Perú

*Autor de Correspondencia, e-mail: henryjavec@outlook.com
} 


\section{INTRODUCCIÓN}

En la actualidad, la problemática ambiental relacionada con la contaminación de los recursos hídricos, pone en riesgo el equilibrio ambiental, la seguridad alimentaria, la salud poblacional y el desarrollo económico sostenible de la población mundial. Según el Informe Mundial de las Naciones Unidas sobre el Desarrollo de los Recursos Hídricos (WWAP, 2018; 2019; 2020; 2021), los países del continente europeo tratarían un $71 \%$ de sus aguas residuales, en Medio Oriente y África del Norte un 51\%, mientras que en América Latina alrededor del 20\%. Se debe indicar, además, que en 32 países de los 48 del continente africano no presentan registro sobre la generación y tratamiento.

En concreto, en el Perú muchos de los cuerpos de agua se encontrarían afectados debido a las descargas de aguas residuales (Gamarra-Torres et al., 2018; Rascón et al., 2021). Por lo que, las entidades reguladoras deberían asumir la responsabilidad para su control, considerando la normativa actual, según sector y los parámetros indicadores de contaminación, que se deben corroborar con los Límites Máximos Permisibles correspondientes, donde los aportes de la industria manufacturera, alimentaria, minera, hidrocarburífera y pesquera son de atención prioritaria (Ayala et al., 2018).

La región San Martin, no es ajena a la realidad de la gran mayoría de ciudades ubicadas en zonas tropicales del Perú y otras de Latinoamérica, pues estas evidencian graves problemas de contaminación de los cuerpos de agua como fuentes receptoras del vertimiento de agua residual urbanas, municipales e incluso industriales sin o escaso tratamiento, lo cual impacta negativamente a su calidad, alterando el ecosistema acuático y conllevando a graves problemas de las poblaciones que lo aprovechan.

Son diversos los factores que conllevan a esta problemática, entre ellas la capacidad de gestión y administrativa por parte de las entidades relacionadas, así como, la escasa investigación por parte de las instituciones públicas y privadas para promover nuevas tecnologías o mejorar las existentes a fin de brindar alternativas de solución viables y sostenibles para el tratamiento de aguas residuales.

La WWAP (2019), menciona que, el aprovechamiento de las aguas residuales cobra gran importancia como fuente alterna de agua; cambiando el paradigma de la gestión de estas, que pasa por un problema para su tratamiento y su posterior vertimiento a contemplar su reutilización y recuperación; cubriendo así la necesidad del agua para la población (agua potable, aguas de riego y agua para la industria); que gracias a una serie de tecnologías emergentes como la electrocoagulación, la cual ofrece ciertas ventajas frente a las tecnologías tradicionales.

En la actualidad, los mataderos "clandestinos", otros en proceso de formalización y los ya formados, siguen vertiendo sus aguas residuales de manera ilegal a los cuerpos de agua o a las redes de alcantarillado. Razón por la cual, la presente investigación busca evaluar las características del agua residual del matadero del distrito de Zapatero inscrito a la provincia de Lamas de la región San Martin y que, mediante electrocoagulación como alternativa ambientalmente amigable mejore sus características respecto a la concentración de materia orgánica a fin de poder ser vertidas sin generar alteraciones significativas.

Bien se sabe que, entre las principales alternativas aplicadas para tratar estas aguas residuales se encuentran las lagunas de oxidación, humedales y los procesos de coagulación química, donde los primeros demandan un tiempo de retención hidráulica relativamente considerable y su tiempo de vida útil es limitado (Butler et al., 2017), mientras que el ultimo hace uso del sulfato de aluminio, lo cual que por ser de origen sintético genera impactos negativos en el ambiente y a la salud de las poblaciones (Krupińska, 2020).

Es ahí en donde radica la importancia de la electrocoagulación, teniendo en cuenta que, no es una tecnología nueva, pero que ha sido poco estudiada y desarrollada; en los últimos años, ha logrado convertirse en la solución para el tratamiento de muchos contaminantes como una técnica limpia comparada con las tecnologías tradicionales, por lo que, su aplicación adecuada permitiría reme- 
diar muchos problemas, que pueda ser una alternativa viable y eficiente sin provocar efectos negativos al ambiente y/o a la salud. Esta investigación tuvo como objetivo evaluar la eficiencia de la electrocoagulación en la remoción de materia orgánica por DBO presente en el agua residual procedente de un matadero.

\section{MATERIALY MÉTODOS}

\section{Lugar de ejecución}

El experimento se desarrolló en el distrito de Morales, provincia y región San Martín, en el laboratorio de la Universidad Peruana Unión ubicada en el Jr. Los Mártires 340 Urb. Santa Lucía - Morales con coordenadas $6^{\circ} 28^{\prime} 21.42^{\prime \prime} \mathrm{S}$ y $76^{\circ} 23^{\prime} 45.88^{\prime \prime} \mathrm{O}$ a una altitud de 271 m.s.n.m., con una temperatura media anual de $27,5^{\circ} \mathrm{C}$ y una precipitación de $988 \mathrm{~mm}$ anuales.

\section{Diseño del sistema}

El sistema de electrocoagulación presentó las siguientes estructuras, una plataforma metálica de $27 \times 22 \times 1.5$ $\mathrm{cm}$, la cual constituye el soporte de un tanque de vidrio con las siguientes dimensiones $15 \times 20 \times 25 \mathrm{~cm}$ (7 500 $\mathrm{mL}$ capacidad volumétrica total) y donde se ancla un soporte universal con una llave nuez, la cual sujeta al cabezal con los electrodos de aluminio (placas de $13 \times 2 \times 02 \mathrm{~cm}$ ) dispuestos en dos filas y con una separación de $5 \mathrm{~cm}$; el soporte universal, además, sujeta a un transformador de corriente eléctrica de 220 voltios -
12 voltios, un regulador de voltaje de 12 y 24 voltios y un switch para encender y apagar el sistema.

\section{Metodología}

La muestra de agua residual de matadero fue colectada durante la etapa de producción, una muestra compuesta, colectada en la cámara de mezcla a diferentes tiempos mientras duro el proceso; luego se transportó en cadena de frio hasta las instalaciones del laboratorio de ingeniería ambiental de la Universidad Peruana Unión - Tarapoto, lugar donde se desarrolló el ensayo. El muestreo y el transporte de la muestra se realizó de acuerdo a lo establecido por APHA, AWWAy WEF (2017).

De la muestra de agua residual, se tomaron $5000 \mathrm{~mL}$ para ser depositados en el tanque de vidrio, posteriormente se montó el cabezal con los electrodos de aluminio, se reguló el sistema eléctrico a 12 voltios y se cronometró por $15 \mathrm{~min}$, concluido este proceso, con una nueva muestra se ensayó a 24 voltios por $10 \mathrm{~min}$. Para ambos casos se realizaron 6 repeticiones, a fin de alcanzar un volumen de muestra que permita cumplir con los volúmenes necesarios para ser remitidos al laboratorio acreditado para el análisis respectivo de los parámetros de interés.

La toma de la muestra para el análisis de los parámetros antes del proceso y después del proceso, como son $\mathrm{pH}$, conductividad eléctrica, demanda bioquímica de oxíge-

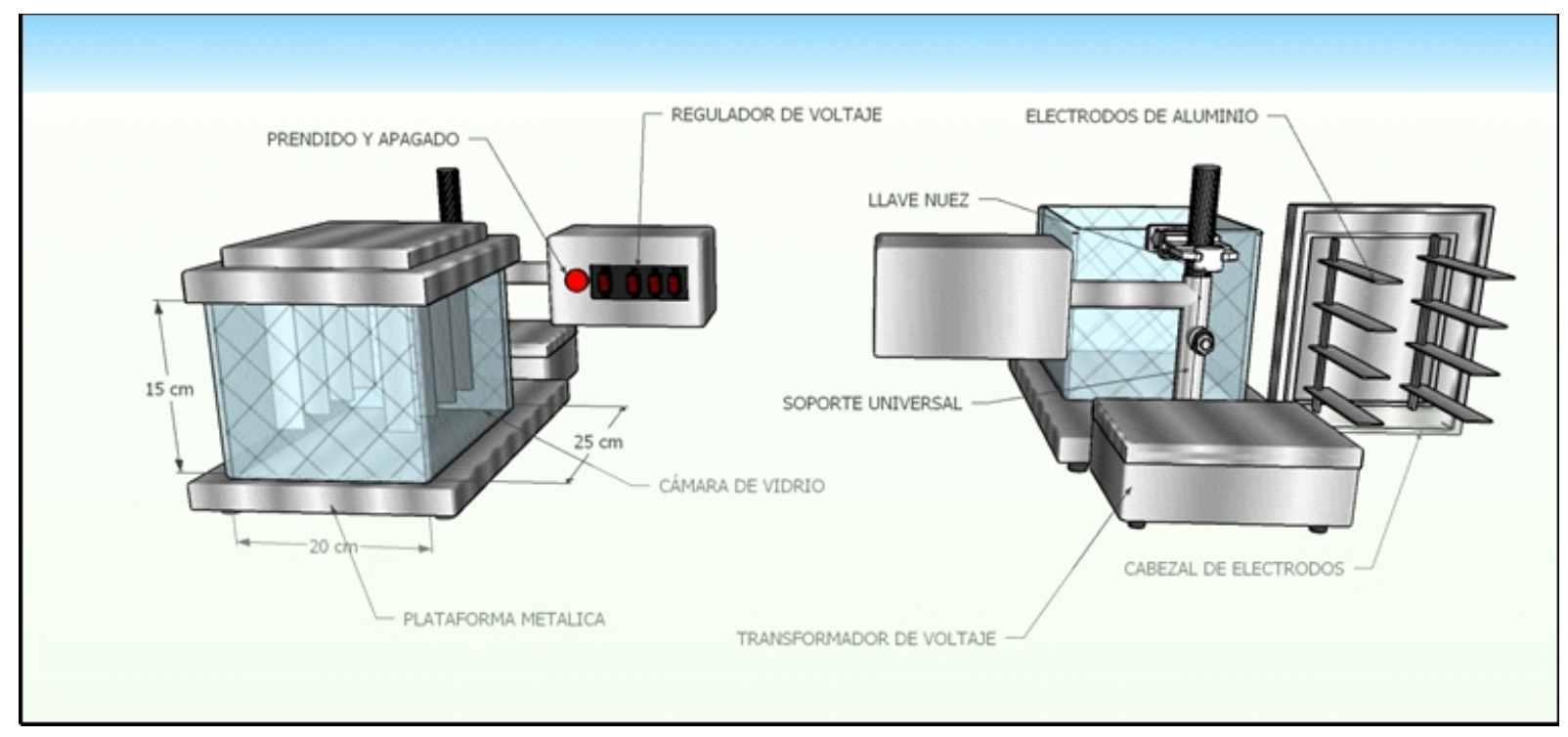

Figura 1. Características del sistema de electrocoagulación. 
no (DBO), solidos suspendidos totales (SST) y turbiedad se realizó en frascos plásticos facilitado por el laboratorio acreditado. Todas las muestras fueron refrigeradas a $4{ }^{\circ} \mathrm{C}$ durante su traslado, para su posterior análisis en el Laboratorio Environmental Quality Analytical Services S.A. (EQUAS S.A.) en Lima.

\section{Análisis de datos}

Los datos fueron sometidos primero a una prueba de normalidad Shapiro-Wilk, al contar con una muestra inferior a 50 (Melo et al., 2020), demostrando que ninguna variable se ajusta a la normal. Posteriormente, se aplicó una prueba Kruskal Wallis (no paramétrica), para determinar si existen diferencias entre los distintos tratamientos. Al existir diferencias, se realizó una prueba de comparaciones múltiples Post-hoc mediante el test de Conover (Zhan et al., 2018; Dul et al., 2020). Todos los análisis estadísticos se realizaron a un nivel de significancia de $\mathrm{p}<0,05$ con el software $\mathrm{R}$ version 3.6.3. ( $\mathrm{R}$ core tema, 2020).

La eficiencia del sistema de electrocoagulación en función a la remoción de la materia orgánica se realizó mediante una fórmula matemática en EXCEL®. Por último, se realizó una comparación con los Límites máximos permisibles (LMP).

\section{RESULTADOS Y DISCUSIÓN}

Todas las variables estudiadas para el tratamiento de aguas residuales con electrocoagulación, demostraron diferencias significativas entre ellos. De los dos tratamientos, el de un voltaje con $24 \mathrm{~V}$, fue el que valores más bajos encontró (Figura 2). Los SST son los que más diferencias muestran entre tratamientos, mientras que el $\mathrm{pH}$ el que menos.

Los valores correspondientes a la eficiencia de remoción de DBO se presentan en la Tabla 1. Donde el segundo tratamiento mediante aplicación de 24 voltios por 10 minutos (E24V) demostró diferencias, respecto al primero con aplicación de 12 voltios por 15 minutos $(\mathrm{E} 12 \mathrm{~V})\left(\chi^{2}=8,516 ; \mathrm{p}=0,003\right)$. La DBO, constituye uno de los agentes que alteran la calidad del agua (Ascón, 2018), teniendo en cuenta que esta proviene de un matadero, en su mayoría estaría constituida por
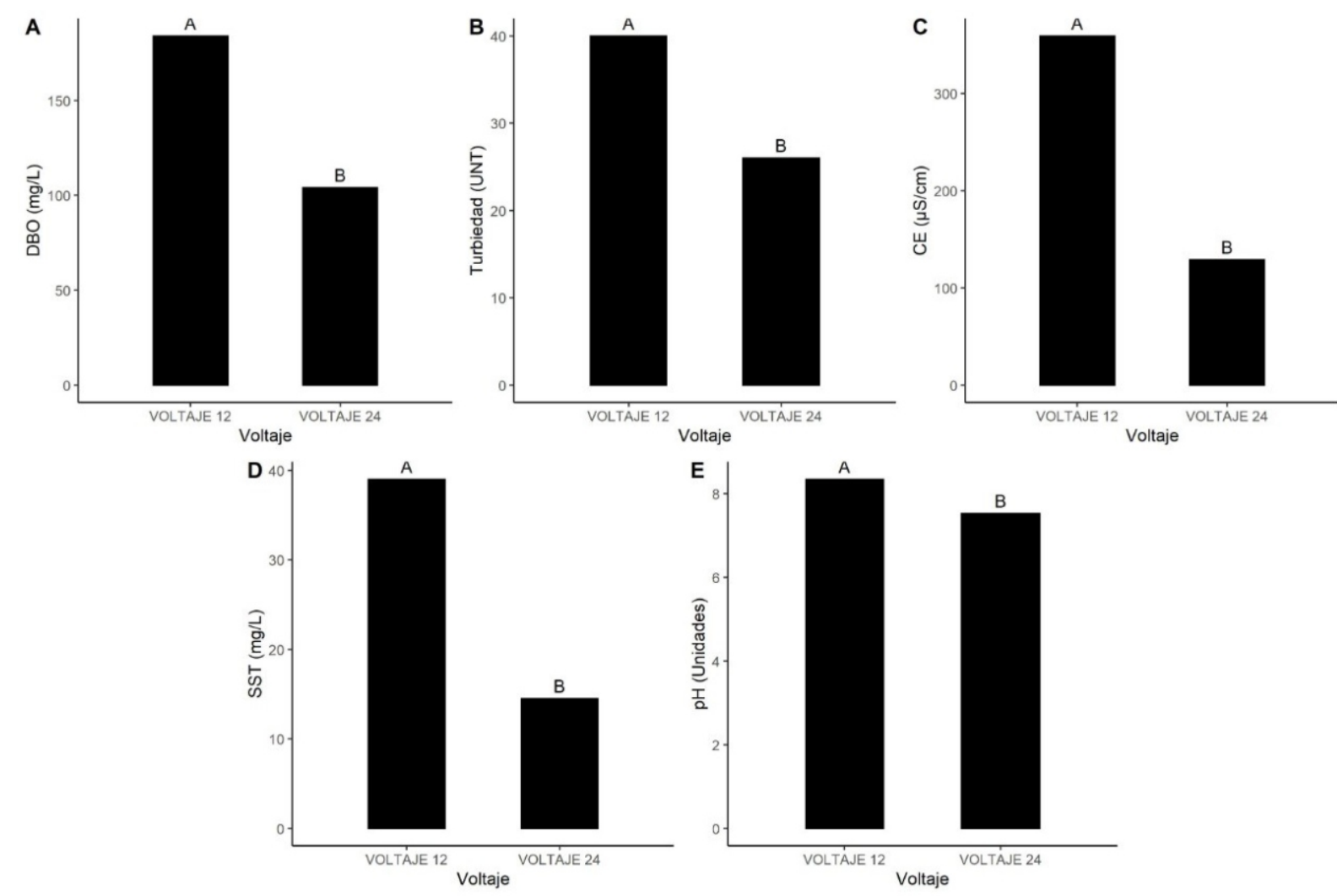

Figura 2. Valores de cada uno de los parámetros lineales. 
carbohidratos (Aleksić, et al., 2020). Gracias a las características polares de los carbohidratos, estos resultan desestabilizados y cambian su configuración electrónica al ser sometidos a una determinada intensidad de corriente (Navarro et al., 2019), promoviendo una reorganización molecular que conlleva a la coagulación final (Aguilar et al., 2020). Los valores de la DBO disminuyeron desde $585 \mathrm{mg} / \mathrm{L}$ hasta $184 \mathrm{mg} / \mathrm{L}$ en el ensayo E12V y 104mg/L en el ensayo E24V, que en términos porcentuales de tratabilidad representa el $68,575 \%$ y $82,194 \%$ respectivamente (Tabla 1 ). Al compararse con otros trabajos, se ven resultados parecidos. El trabajo de Rusdianasari et al. (2021), utilizaron variaciones de flujo eléctrico de 12,15 y $18 \mathrm{~V}$ y tiempos de retención de 30, 60, 90, 120 y $150 \mathrm{~min}$, alcanza una mayor eficiencia con $18 \mathrm{~V}$ y un tiempo de $150 \mathrm{~min}$. Sin embargo, el voltaje puede ser variable dependiendo de varios factores, principalmente las características fisicoquímicas de las moléculas que constituyen al DBO (Hawari et al., 2020).

El tratamiento de las aguas residuales, independiente de la actividad productiva que lo genera asegura la calidad de agua del cuerpo receptor, su disponibilidad y aprovechamiento de manera sostenible (Morsy et al., 2020). Estos compuestos orgánicos afectan directa o indirectamente los servicios eco-sistémicos que los cuerpos de agua brindarían (Chen et al., 2021). Es así, actualmente, cada país evalúa y fiscaliza los vertimientos de las aguas residuales. En el Perú, el Organismo de Evaluación y Fiscalización Ambiental (OEFA), señala que, las aguas residuales que no presenten tratamiento o este sea inadecuado, alteran la tratabilidad por sobrecarga en las plantas de tratamiento, lo cual genera que los efluentes tratados excedan los Limites correspondientes (OEFA, 2014).
El primer tratamiento (E12V), tiene un menor porcentaje de remoción en función de la turbiedad, conductividad eléctrica $\left(\chi^{2}=8,516 ; p=0,003\right)$. Esto permite explicar que los coloides que constituyen la turbiedad inicial (444 UNT), no lograron ser desestabilizados suficientemente mediante la aplicación de 12 voltios por 15 minutos, alcanzando una turbiedad final de 39,67 UNT (91,066 \% de eficiencia) respecto al segundo tratamiento (E24V) donde se alcanzó 26 UNT (eficiencia del 94,144\%) (Tabla 1), esto guarda relación directa con lo descrito por Sun, et al., (2019), que para sedimentar los coloides requiere de una energía y un tiempo suficiente capaz de desestabilizarlos, constituyan el floc adecuado y su posterior sedimentación. Recalcando que, electro-coagulación utiliza una fuente de poli electrodos que aportan iones desestabilizadores de las partículas coloidales, semejante a la acción de los coagulantes utilizados en el tratamiento convencional (Yasri et al., 2020).

La materia orgánica soluble presente en el agua residual (Meyo et al., 2021), tiene influencia directa sobre la conductividad eléctrica, por lo que resulta importante su evaluación en la determinación de la calidad del agua (Han et al., 2021). Al realizar la evaluación de la conductividad eléctrica, esta disminuye desde 1579 $\mu \mathrm{S} / \mathrm{cm}$ hasta $359,35 \mu \mathrm{S} / \mathrm{cm}$ en el tratamiento E12V y $129 \mu \mathrm{S} / \mathrm{cm}$ en el tratamiento $\mathrm{E} 24 \mathrm{~V}\left(\chi^{2}=8,366 ; \mathrm{p}=\right.$ $0,004)$, lo cual representa una eficiencia en la remoción del 77,230\% y 91,836\% respectivamente(Tabla 1).

La materia orgánica soluble presente en el agua residual resulta ser un problema grave si no se trata $\mathrm{y}$ alcanza cuerpos de agua lenticos, ya que, estos compuestos pueden ser asimilados fácilmente y desencadenarían un proceso de eutrofización gracias el alto contenido de compuestos nitrogenados y azufrados

Tabla 1. Eficiencia de la remoción en agua residual de matadero

\begin{tabular}{lcc}
\hline Parámetro & \multicolumn{2}{c}{ Eficiencia (\%) } \\
& E12V & E24V \\
\hline DBO & 68,575 & 82,194 \\
Turbiedad & 91,066 & 94,144 \\
Conductividad Eléctrica & 77,239 & 91,836 \\
SST & 93,813 & 96,888 \\
\hline
\end{tabular}


(Smolet al., 2020).

Los sólidos suspendidos totales (SST) presentes en el agua residual de matadero está constituida básicamente por las excretas, sangre, grasas, pelos y otros que se desprenden o se generan en las actividades propias de cada una de las etapas del proceso (Ziara et al., 2018). Algunos de estos elementos, dependiendo del volumen y peso molecular, logran sedimentar fácilmente, mientras que otros pueden flotar o en el peor de los casos pueden quedar en suspensión por un tiempo mayor y pasar a constituir los denominados colides (AlJaberi et al., 2020).

Los SST en la presente evaluación se lograron disminuir desde los $466 \mathrm{mg} / \mathrm{L}$ hasta $29 \mathrm{mg} / \mathrm{L}$ en el tratamiento E12V y hasta $14.5 \mathrm{mg} / \mathrm{L}$ en el tratamiento E24V $\left(\chi^{2}=\right.$ $8,516 ; p=0,003)$. Esto correspondería a una eficiencia en la remoción del 93,813\% y 96,888\% respectivamente. Dicha eficiencia, permite corroborar lo visto en los trabajos realizados por Reátegui et al. (2020) y Chakchouk et al. (2017), donde emplearon la electrocoagulación para tratar agua residual de matadero, teniendo en cuenta el voltaje y el tiempo de retención hidráulica. Ambos obtuvieron una tratabilidad mayor del 50\%, dependiendo del parámetro indicador analizado.

Es importante, tener en cuenta que en toda evaluación se debe realizar un monitoreo de los parámetros básicos, donde se tiene que incluir el $\mathrm{pH}$, ya que estos pueden permitir una mejor interpretación del proceso en sí. Por ejemplo, Oliveira et al., (2020), señala que el $\mathrm{pH}$ en los procesos donde se aplica electrocoagulación es muy variable. Así, el pH vario desde 11,77 hasta $8,345(12 \mathrm{~V})$ y $7,525(24 \mathrm{~V})$, lo que permite interpretar que si se diera el caso donde el agua post tratamiento se vierte a un cuerpo de agua, esta aplicaría según la normativa nacional actual. Siendo más eficiente el tratamiento con $24 \mathrm{~V}\left(\chi^{2}=8,516 ; \mathrm{p}=0,003\right)$.

\section{CONCLUSIONES}

La aplicación del sistema de electrocoagulación con electrodos de aluminio a $12 \mathrm{~V}$ por 15 minutos y $24 \mathrm{~V}$ por 10 minutos permitieron reducir significativamente los valores de la conductividad eléctrica, DBO, soli- dos suspendidos totales (SST) y la Turbiedad. Por tanto, la electrocoagulación es una alternativa viable para tratar el agua residual procedente de matadero, donde la utilización de electrodos de aluminio con una intensidad de 12 y 24 voltios por 15 y 10 minutos respectivamente, es eficiente $(>90 \%)$ para tratar a la materia orgánica en función de la DBO. El agua residual procedente del proceso de matadero tratada con esta tecnología podría ser utilizadas para riego agrícola o vertidas a un cuerpo receptor sin ocasionar impacto ambiental significativo. Se recomienda realizar ensayos experimentales con otros voltajes y otros tiempos de retención hidráulica.

\section{V.AGRADECIMIENTOS}

Al personal administrativo de la municipalidad del distrito de Zapatero, por facilitarnos el acceso al Matadero Municipal del mismo distrito, provincia de Lamas y región San Martin.

\section{REFERENCIAS BIBLIOGRÁFICAS}

Aguilar, E., L. Marrufo, y W. Neyra. 2020. “Efficiency of Electrocoagulation Method to Reduce COD, BOD and TSS in Tannery Industry Wastewater: Application of the Box-Behnken Design." Leather and Footwear Journal 20 (3): 217-28. DOI:10.24264/1fj.20.3.1.

Aleksić, N., A. Nešović, V. Šušteršič, D. Gordić, y D. Milovanović. 2020. "Slaughterhouse Water Consumption and Wastewater Characteristics in the Meat Processing Industry in Serbia." Desalination and Water Treatment 190: 98-112. DOI:10.5004/dwt.2020.25745.

AlJaberi, F., S. Ahmed, y H. Makki. 2020. "Electrocoagulation Treatment of High Saline Oily Wastewater: Evaluation and Optimization."Heliyon 6 (6): e 03988 . DOI:10.1016/j.heliyon.2020.e03988.

APHA, AWWA, y WEF. 2017. Standard Methods for the Examination of Water and Wastewater. Washington D.C. (EEUU): American Public Health Association/American Water Works 
Association/Water Environment Federation.

Ascón, E. 2018. "Elimination of Chemical Oxygen Demand from Domestic Residual Water by Electrocoagulation with Aluminum and Iron Electrodes." Ambiente e Agua - An Interdisciplinary Journal of Applied Science 13 (5): 1. DOI:10.4136/ambi-agua.2240.

Ayala, Y. R., E. Calderón, J. Rascón, y R. Collazos. 2018. "Fitorremediación de Aguas Residuales Domésticas Utilizando Las Especies Eichhornia Crassipes, Nymphoides Humboldtiana y Nasturtium Officinal." Revista de Investigación en Agroproducción Sustentable 2 (3): 48-53. DOI: 10.25127/aps.20183.403

Butler, E., Y. T. Hung, M. S. A. Ahmad, R. Yu-Li Yeh, R. Lian-Huey Liu, y Y. P. Fu. 2017. “Oxidation Pond for Municipal Wastewater Treatment." Applied Water Science 7 (1): 31-51. DOI:10.1007/s13201-015-0285-z.

Chakchouk, I., N. Elloumi, C. Belaid, S. Mseddi, L. Chaari, y M. Kallel. 2017. “A Combined Electrocoagulation-Electrooxidation Treatment for Dairy Wastewater." Brazilian Journal of Chemical Engineering 34 (1): 109-17. D O I : $1 \begin{array}{llllllllllll} & 0 & \text {. } & 5 & 9 & 0 & / & 0 & 1 & 0 & 4 & -\end{array}$ 6632.20170341s20150040.

Chen, X., Y. Wang, T. Sun, Y. Huang, Y. Chen, M. Zhang, y C. Ye. 2021. "Effects of Sediment Dredging on Nutrient Release and Eutrophication in the Gate-Controlled Estuary of Northern Taihu Lake." Journal of Chem$i s t r y \quad 20021 \quad$ ( 1 ): $1-13$. DOI:10.1155/2021/7451832.

Dul, J., E. van der Laan, y R. Kuik. 2020. “A Statistical Significance Test for Necessary Condition Analysis." Organizational Research Methods $23 \quad(2): \quad 385-95$. DOI:10.1177/1094428118795272.

Gamarra-Torres, O. A., M. A. Barrena-Gurbillón, E. Barboza-Castillo, J. Rascon, F. Corroto, y L. A. Taramona-Ruiz. 2018. "Fuentes de Contaminación Estacionales En La Cuenca Del Río
Utcubamba , Región Amazonas , Perú.” Arnaldoa 25 (1): 179-94.

Han, Z., M. Xiao, F. Yue, Y. Yi, y K. Mostofa. 2021. "Seasonal Variations of Dissolved Organic Matter by Fluorescent Analysis in a Typical River Catchment in Northern China." Water 13 (4): 494. DOI:10.3390/w13040494.

Hawari, A., A. Alkhatib, M. Hafiz, y P. Das. 2020. “A Novel Electrocoagulation Electrode Configuration for the Removal of Total Organic Carbon from Primary Treated Municipal Wastewater." Environmental Science and Pollution Research 27 (19): 23888-98. DOI:10.1007/s11356-020-08678-4.

Krupińska, I. 2020. “Aluminium Drinking Water Treatment Residuals and Their Toxic Impact on Human Health." Molecules 25 (3): 641. DOI:10.3390/molecules25030641.

Melo, O. O., L. A. López, y S. E. Melo. 2020. Diseño de Experimentos - Métodos y Aplicaciones. Bogotá (Colombia): Universidad Nacional de Colombia Facultad de Ciencias. DOI:10.36385/FCBOG-4-0.

Meyo, H. B., M. Njoya, M. Basitere, S. K. Obed Ntwampe, y E. Kaskote. 2021. "Treatment of Poultry Slaughterhouse Wastewater (PSW) Using a Pretreatment Stage, an Expanded Granular Sludge Bed Reactor (EGSB), y a Membrane Bioreactor (MBR).” Membranes $11 \quad$ ( 5 ) : $\quad 345$. DOI:10.3390/membranes11050345.

Morsy, K., A. Mishra, y M. Galal. 2020. “Water Quality Assessment of the Nile Delta Lagoons." Air, Soil and Water Research 13 (1): $\begin{array}{llllllllllllllll}1 & 1 & 7 & 8 & 6 & 2 & 2 & 1 & 2 & 0 & 9 & 6 & 3 & 0 & 7\end{array}$. DOI: $10.1177 / 1178622120963072$.

Navarro, D., J. Abelilla, y H. Stein. 2019. "Structures and Characteristics of Carbohydrates in Diets Fed to Pigs: A Review." Journal of Animal Science and Biotechnology 10 (1): 39. DOI:10.1186/s40104-019-0345-6.

Oliveira, A. D., J. Ribeiro, E. Neto, A. de Lima, Á. Ama- 
zonas, L. da Silva, y R. do Nascimento. 2020.

"Removal of Natural Organic Matter from Aqueous Solutions Using Electrocoagulation Pulsed Current: Optimization Using Response Surface Methodology." Water Science and Technology 82 ( 1 ): 56-66. DOI:10.2166/wst.2020.323.

OEFA (Organismo de Evaluación y Fiscalización Ambiental). 2014. Fiscalización Ambiental En Aguas Residuales. Lima (Perú): MINAM.

R Core Team. 2013. R: A language and environment for statistical computing. R Foundation for Statistical Computing, Vienna, Austria. http://www.R-project.org/.

Rascón, J., F. Corroto, D. Leiva-Tafur, y O. A. Gamarra Torres. 2021. "Variaciones Limnológicas Espaciotemporales de Un Lago Altoandino Tropical Al Norte de Perú." Ecología Austral 31 (2): 343-56. DOI:10.25260/ea.21.31.2.0.1200.

Reátegui, W., S. Tuesta, C. Ochoa, J. Huamán, M. King, E. F. Estrada, W. Bulege-Gutierrez, R. A. YuliPosadas, y V. Fernández-Guzmán. 2020. "Electrocoagulation in Batch Mode for the Removal of the Chemical Oxygen Demand of an Effluent from Slaughterhouse Wastewater in Lima Peru: Fe and Al Electrodes." Desalination and Water Treatment 201 (1): 206-18. DOI:10.5004/dwt.2020.26175.

Rusdianasari, Y. B., A. Syakdani, y D. Mayasari. 2021. "The Effectiveness of Electrocoagulation Process in Rubber Wastewater Treatment Using Combination Electrodes.” IOP Conference Series: Earth and Environmental Science 709 ( 1 ). D O I : $10.1088 / 1755$ 1315/709/1/012009.

Smol, M., M. Preisner, A. Bianchini, J. Rossi, L. Hermann, T. Schaaf, J. Kruopienè, et al. 2020. "Strategies for Sustainable and Circular Management of Phosphorus in the Baltic Sea Region: The Holistic Ppproach of the InPhos Project." Sustainability 12 (6): 2567. DOI:10.3390/su12062567.
Sun, Y., S. Zhou, P. C. Chiang, y K. Shah. 2019. “Evaluation and Optimization of Enhanced Coagulation Process: Water and Energy Nexus." WaterEnergy Nexus 2 (1): 25-36. DOI:10.1016/j.wen.2020.01.001.

WWAP (Programa Mundial de Evaluación de los Recursos Hídricos de la UNESCO). 2018. Informe Mundial de las Naciones Unidas sobre el Desarrollo de los Recursos Hídricos 2018: Soluciones basadas en la naturaleza para la gestión del agua. París (Francia): UNESCO.

WWAP (Programa Mundial de Evaluación de los Recursos Hídricos de la UNESCO). 2019. Informe Mundial de las Naciones Unidas sobre el Desarrollo de los Recursos Hidricos 2019: No dejar a nadie atrás. París (Francia): UNESCO.

WWAP (Programa Mundial de Evaluación de los Recursos Hídricos de la UNESCO). 2020. Informe Mundial de las Naciones Unidas sobre el Desarrollo de los Recursos Hídrico 2020: Agua y Cambio Climático. París (Francia): UNESCO.

WWAP (Programa Mundial de Evaluación de los Recursos Hídricos de la UNESCO). 2021. Valuing Water. París (Francia): UNESCO.

Yasri, N., J. Hu, M. G. Kibria, y E. Roberts. 2020. "Electrocoagulation Separation Processes." En Multidisciplinary Advances in Efficient Separation Processes ACS (ed) Washington D. C. (EEUU): ACS. DOI:10.1021/bk-20201348.ch006.

Zhang, J., Y. Wang, Y. Zhao, y X. Cai. 2018. “Applications of Inferential Statistical Methods in Library and Information Science." Data and Information Management 2 (2): 103-20. DOI:10.2478/dim-2018-0007.

Ziara., Rami, S. Li, J. Subbiah, y B. Dvorak. 2018. "Characterization of Wastewater in Two U.S. Cattle Slaughterhouses." Water Environment Research 90 (9): $851-63$. DOI:10.2175/106143017X15131012187971. 